\title{
SCIENTIFIC REPORTS

\section{OPEN Increased circulating galectin-1 levels are associated with the progression of kidney function decline in patients undergoing coronary angiography}

\author{
Chin-Sung Kuo $0^{1,2,3}$, Ruey-Hsing Chou ${ }^{2,3,4,5}$, Ya-Wen Lu ${ }^{2,4}$, Yi-Lin Tsai ${ }^{2,4}$, Po-Hsun Huang ${ }^{2,3,4,5^{*}}$ \& \\ Shing-Jong $\operatorname{Lin}^{2,3,4,6,7 *}$
}

Galectin-1 modulates acute and chronic inflammation, and is associated with glucose homeostasis and chronic renal disease. Whether the serum galectin-1 level can predict short-term and longterm renal outcomes after contrast exposure in patients undergoing coronary angiography (CAG) remains uncertain. This study aimed to evaluate the relationship between the serum galectin-1 level and the incidence of contrast-induced nephropathy (CIN), and to investigate the predictive role of the circulating galectin-1 level for renal function decline in patients undergoing CAG. In total, 798 patients who had undergone CAG were enrolled. Baseline creatinine and serum galectin-1 levels were determined before CAG. CIN was defined as an increase in the serum creatinine level of $0.5 \mathrm{mg} / \mathrm{dl}$ or a $25 \%$ increase from baseline within $48 \mathrm{~h}$ after the procedure, and renal function decline was defined as $>30 \%$ reduction of the estimated glomerular filtration rate from baseline. All patients were followed for at least 1 year or until the occurrence of death after CAG. Overall, CIN occurred in 41 (5.1\%) patients. During a median follow-up period of $1.4 \pm 1.1$ years, $80(10.0 \%)$ cases showed subsequent renal function decline. After adjustment for demographic characteristics, kidney function, traditional risk factors, and medications, higher galectin-1 levels were found to be associated independently with a greater risk of renal function decline [tertile 2: hazard ratio (HR) 5.56, 95\% confidence interval (CI) 1.79-17.22; tertile 3: $\mathrm{HR} 5.56,95 \% \mathrm{Cl} 1.97-16.32$ ], but not with $\mathrm{CIN}$, regardless of the presence of diabetes. In conclusion, higher baseline serum galectin-1 levels were associated with a greater risk of renal function decline in patients undergoing CAG, but were not associated independently with CIN.

Chronic kidney disease (CKD) is a serious public health problem, and its incidence and prevalence are increasing ${ }^{1}$. The early detection of individuals at risk of CKD development or progression is especially important, as early-stage $\mathrm{CKD}$ is prevalent and contributes greatly to cardiovascular disease. Research clearly indicates that patients with coronary artery disease (CAD) are at increased risk of progressive renal dysfunction ${ }^{2}$. These patients may be further exposed to the risk of contrast-induced nephropathy (CIN), which has an incidence rate of 4.4-22.1\%, if they undergo coronary angiography $(\mathrm{CAG})^{3}$. CIN has traditionally been considered to be a benign and reversible disease, but it may prolong hospital stays and increase in-hospital mortality ${ }^{4}$. Accumulating evidence suggests that CIN is related to long-term renal function decline, in addition to short-term outcomes ${ }^{5,6}$. Efforts to predict the occurrence of CIN include the development of risk scoring systems composed of baseline risk factors ${ }^{7}$, renal

\footnotetext{
${ }^{1}$ Division of Endocrinology and Metabolism, Department of Medicine, Taipei Veterans General Hospital, Taipei, Taiwan. ${ }^{2}$ Cardiovascular Research Center, National Yang-Ming University, Taipei, Taiwan. ${ }^{3}$ Institute of Clinical Medicine, National Yang-Ming University, Taipei, Taiwan. ${ }^{4}$ Division of Cardiology, Department of Medicine, Taipei Veterans General Hospital, Taipei, Taiwan. ${ }^{5}$ Department of Critical Care Medicine, Taipei Veterans General Hospital, Taipei, Taiwan. ${ }^{6}$ Healthcare and Services Center, Taipei Veterans General Hospital, Taipei, Taiwan. ${ }^{7}$ Taipei Heart Institute, Taipei Medical University, Taipei, Taiwan. *email: huangbsvgh@gmail.com; sjlin@vghtpe.gov.tw
} 


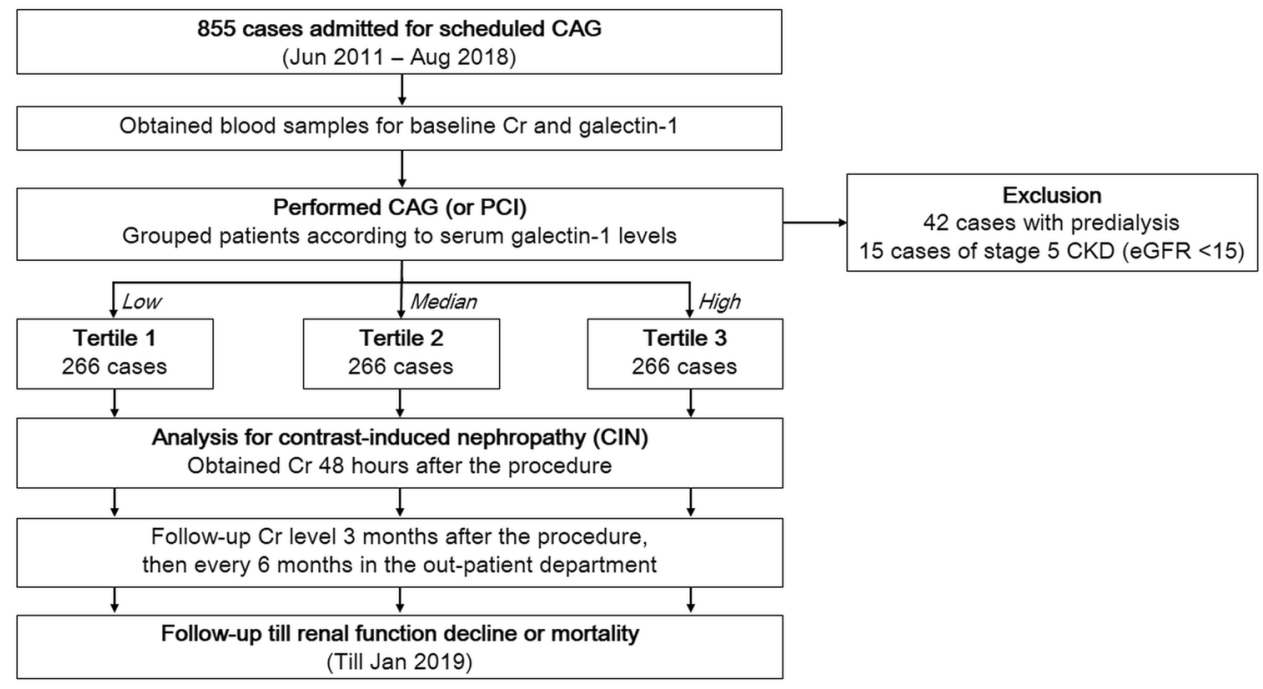

Figure 1. Flowchart of patient recruitment and follow-up.

Doppler evaluation $^{8}, 24$-h serum creatinine elevation ${ }^{9}$, and renal injury biomarkers ${ }^{10-14}$. However, no predictive marker for long-term renal outcomes in patients undergoing CAG has been established.

Lectin-glycan interactions have been reported to be regulators of extensive physiological and pathological processes. Galectins form a group of proteins that can bind to $\beta$-galactoside sugars by $\mathrm{N}$ - or O-linked glycosylation through their carbohydrate recognition domains ${ }^{15}$. Galectins comprise three categories: monomeric, chimeric, and tandem-repeat types. They are characterized as homodimers of $14-\mathrm{kDa}$ subunits with two $\beta$-galactoside-binding sites, and are expressed in many tissues ${ }^{15}$. Fifteen galectin isoforms, numbered in order of discovery (galectin-1-15), are recognized ${ }^{15}$. Galectin-1 is found in cytoplasm and on cell surfaces, and can be secreted to the extracellular matrix ${ }^{15}$. It modulates cell signaling, proliferation, and survival, and contributes to the control of inflammation and neovascularization ${ }^{16}$.

Diabetes is a traditional risk factor for $\mathrm{CIN}^{7}$ and $\mathrm{CKD}^{17}$. Recent evidence indicates that galectin-1 levels are increased in the proteome in subcutaneous interstitial fluid ${ }^{18}$ and plasma ${ }^{19}$ in patients with type 2 diabetes. The galectin-1 level is also elevated in the glomerular proteome in mice with $\mathrm{CKD}^{20}$. An in vitro study implicated galectin-1 in diabetic nephropathy, as seen in human podocytes in high-glucose culture ${ }^{21}$. Furthermore, galectin-1 was reported to be a new fibrosis protein and potential treatment target in the context of diabetic nephropathy ${ }^{22}$. However, whether the serum galectin-1 level can predict CIN and kidney function decline in patients undergoing CAG remains unclear. This study aimed to evaluate the relationship between the serum galectin-1 level and the incidence of CIN, and to clarify the predictive role of galectin-1 in renal function decline, in patients with suspected CAD undergoing CAG at a single hospital. We hypothesized that elevated galectin-1 levels, which have been reported to be associated with diabetes and diabetic nephropathy, would also be predictive markers of CIN and renal function decline in these patients. We also sought to clarify whether the possible predictive role of galectin-1 would be independent of diabetes.

\section{Results}

Baseline patient characteristics. In total, 798 subjects who underwent elective CAG and/or percutaneous coronary intervention (PCI) were enrolled in this analysis (Fig. 1). The mean age of the study population was $67 \pm 12$ years, and $67.5 \%$ of patients were male. Table 1 summarizes the clinical and demographic characteristics of the patients, grouped by galectin-1 concentration. Patients with higher plasma galectin-1 concentrations were older and had higher incidences of hypertension, diabetes, CKD, heart failure, and multiple vessel disease. Subjects in the highest galectin-1 tertile had significantly increased levels of fasting glucose, proteinuria, and decreased hemoglobin levels, estimated glomerular filtration rates (eGFRs), and left ventricular ejection fractions (LVEFs).

Kaplan-Meier survival analysis was performed to investigate the potential impact of the baseline galectin-1 level on adverse event-free survival (Fig. 2). Patients in the highest galectin-1 group were at significantly greater risk of renal function decline than were those in the lowest galectin-1 group $(p<0.001)$. Patients with prior CIN were also at significantly greater risk of renal function decline than were those without prior $\operatorname{CIN}(p<0.001)$.

Baseline characteristics according to the presence of diabetes. Of the 798 study subjects, 275 (34.5\%) were diabetic. Table 2 shows the clinical and demographic characteristics of patients grouped by the presence of diabetes mellitus. Patients with diabetes were older and had higher incidences of hypertension, CKD, and peripheral arterial disease. In addition to anti-diabetic agents, diabetic patients also received more antiplatelet therapy, statins, and anti-hypertensive agents. Compared with nondiabetic patients, patients with diabetes had higher levels of serum galectin-1, more proteinuria, lower hemoglobin and total cholesterol levels, and reduced LVEFs. 


\begin{tabular}{|c|c|c|c|c|}
\hline Characteristic & $\begin{array}{l}\text { Tertile } 1(\mathrm{n}=\mathbf{2 6 6}) \\
\text { Galectin } \mathbf{1}<\mathbf{1 6 . 0}\end{array}$ & $\begin{array}{l}\text { Tertile } 2(n=266) \\
\text { Galectin 1: 16.1-22.2 }\end{array}$ & $\begin{array}{l}\text { Tertile } 3(n=266) \\
\text { Galectin } 1 \geq 22.2\end{array}$ & $P$ \\
\hline Age (years) & $61(54-71)$ & $67(60-75)$ & $73.0(62-81)$ & $<0.001$ \\
\hline Male, n (\%) & $180(67.7)$ & $174(65.4)$ & $185(69.5)$ & 0.594 \\
\hline Smoking, n (\%) & $75(28.2)$ & $91(34.2)$ & $99(37.2)$ & 0.080 \\
\hline BMI $\left(\mathrm{kg} / \mathrm{m}^{2}\right)$ & $24.8(22.4-27.6)$ & $25.8(23.8-28.5)$ & $25.7(22.9-28.2)$ & 0.019 \\
\hline \multicolumn{5}{|l|}{ Medical history, $n(\%)$} \\
\hline Hypertension & $148(55.6)$ & $175(65.8)$ & $199(74.8)$ & $<0.001$ \\
\hline Diabetes & $70(26.3)$ & $86(32.3)$ & $119(44.7)$ & $<0.001$ \\
\hline Chronic kidney disease & $5(1.9)$ & $5(1.9)$ & $39(14.7)$ & $<0.001$ \\
\hline Heart failure & $9(3.4)$ & $9(3.4)$ & $33(12.4)$ & $<0.001$ \\
\hline Peripheral arterial disease & $18(6.8)$ & $12(4.5)$ & $31(11.7)$ & 0.007 \\
\hline Previous stroke & $12(4.5)$ & $12(4.5)$ & $22(8.3)$ & 0.101 \\
\hline \multicolumn{5}{|l|}{ Medications, $n(\%)$} \\
\hline Antiplatelet agents & $136(51.1)$ & $136(51.1)$ & $152(57.1)$ & 0.276 \\
\hline ACEi/ARB & $61(22.9)$ & $80(30.1)$ & $92(34.6)$ & 0.012 \\
\hline Diuretics & $10(3.8)$ & $26(9.8)$ & $36(13.2)$ & 0.001 \\
\hline Oral antidiabetic agents & $41(15.4)$ & $50(18.8)$ & $57(21.4)$ & 0.202 \\
\hline Insulin & $9(3.4)$ & $13(4.9)$ & $23(8.6)$ & 0.025 \\
\hline Statins & $84(31.6)$ & $90(33.8)$ & $71(26.7)$ & 0.189 \\
\hline \multicolumn{5}{|l|}{ Laboratory data } \\
\hline White blood cells (K/cumm) & $6.6(5.5-7.6)$ & $6.7(5.7-7.8)$ & $6.9(5.8-7.9)$ & 0.195 \\
\hline Hemoglobin (g/dL) & $13.5(12.5-14.3)$ & $13.4(12.4-14.2)$ & $12.8(11.3-14.0)$ & $<0.001$ \\
\hline Fasting glucose (mg/dL) & $97.5(87-119)$ & $98.0(89-114)$ & $101(91-128)$ & 0.044 \\
\hline $\mathrm{HbAlc}(\%)$ & $6.5(6.0-7.3)$ & $6.3(5.8-7.4)$ & $6.5(5.9-7.3)$ & 0.419 \\
\hline Proteinuria $(\mathrm{mg} / \mathrm{dL})$ & $0.0(0.0-0.0)$ & $0.0(0.0-0.0)$ & $0.0(0.0-15.0)$ & $<0.001$ \\
\hline Proteinuria $\geq 30 \mathrm{mg} / \mathrm{dL}, \mathrm{n}(\%)$ & $10(3.8)$ & $15(5.7)$ & $53(20.3)$ & $<0.001$ \\
\hline eGFR $\left(\mathrm{ml} / \mathrm{min} / 1.73 \mathrm{~m}^{2}\right)$ & $80.5(68-9)$ & $70.7(59-81)$ & $56.9(42-73)$ & $<0.001$ \\
\hline Total cholesterol (mg/dL) & $160(143-182)$ & $159(137-182)$ & $159(137-182)$ & 0.487 \\
\hline Galectin 1 (ng/mL) & $13.3(10.2-14.6)$ & $18.4(17.3-20.0)$ & $27.7(24.5-32.6)$ & $<0.001$ \\
\hline \multicolumn{5}{|l|}{ Cardiac catheterization } \\
\hline Mean blood pressure $(\mathrm{mmHg})$ & $105(95-113)$ & $105(92-115)$ & $108(98-118)$ & 0.281 \\
\hline LVEF (\%) & $59(54-64)$ & $60(54-64)$ & $57(52-62)$ & 0.002 \\
\hline Significant CAD, n (\%) & $113(43)$ & $135(51)$ & $152(57)$ & 0.003 \\
\hline Underwent PCI, n (\%) & $111(42)$ & $124(47)$ & $120(45)$ & 0.509 \\
\hline Contrast volume (ml) & $50(50-95)$ & $50(50-180)$ & $50(50-225)$ & 0.002 \\
\hline \multicolumn{5}{|l|}{ Outcomes } \\
\hline CIN, n (\%) & $7(2.6)$ & $12(4.5)$ & $22(8.3)$ & 0.011 \\
\hline eGFR decline > 30\%, n (\%) & $7(2.6)$ & $20(7.5)$ & $53(19.9)$ & $<0.001$ \\
\hline All-cause mortality, n (\%) & $4(1.5)$ & $1(0.4)$ & $8(3.0)$ & 0.055 \\
\hline
\end{tabular}

Table 1. Baseline characteristics of the study cohort by tertiles of the serum galectin-1 concentration. BMI, body mass index; ACEi/ARB, angiotensin-converting enzyme inhibitor/angiotensin receptor blocker; HbA1c, glycosylated hemoglobin Alc; eGFR, estimated glomerular filtration rate; LVEF, left ventricular ejection fraction; CAD, coronary artery disease; PCI, percutaneous coronary intervention; CIN, contrast-induced nephropathy.

Independent correlates of contrast-induced nephropathy and predictors of renal function decline. In univariate logistic regression analysis, a higher galectin-1 level, older age, history of diabetes, lower hemoglobin level, decreased baseline eGFR, and presence of proteinuria were associated significantly with a greater risk of CIN. To identify independent predictors of CIN, multivariable logistic regression analysis was performed. After adjustment for age, sex, and baseline eGFR, the highest serum galectin-1 level was associated significantly with CIN [odds ratio (OR), 2.64; $95 \%$ confidence interval (CI), 1.03-6.76; $p=0.04$ ]. After adjustment for age, sex, and all factors that were significant in the univariate analysis, the serum galectin-1 level was not associated significantly with CIN, but associations persisted with age (OR, 1.04; 95\% CI, 1.01-1.08; $p=0.026$ ), and the hemoglobin level (OR, 0.67; 95\% CI, 0.54-0.83; $p<0.001$; Table 3).

In a univariate Cox regression analysis, older age, histories of hypertension and diabetes, proteinuria, higher serum galectin-1 level, lower hemoglobin level, reduced eGFR, and lower LVEF, as well as prior CIN, were associated significantly with a higher incidence of renal function decline. In a multivariable Cox regression analysis, the circulating galectin-1 level [tertile 2: hazard ratio (HR) 5.56, 95\% CI 1.79-17.22; tertile 3: HR 5.67, 95\% CI 1.97-16.32), hemoglobin level (HR, $0.83 ; 95 \%$ CI, $0.71-0.98 ; p=0.025)$ and proteinuria (HR, $1.00 ; 95 \% \mathrm{CI}$, 
A

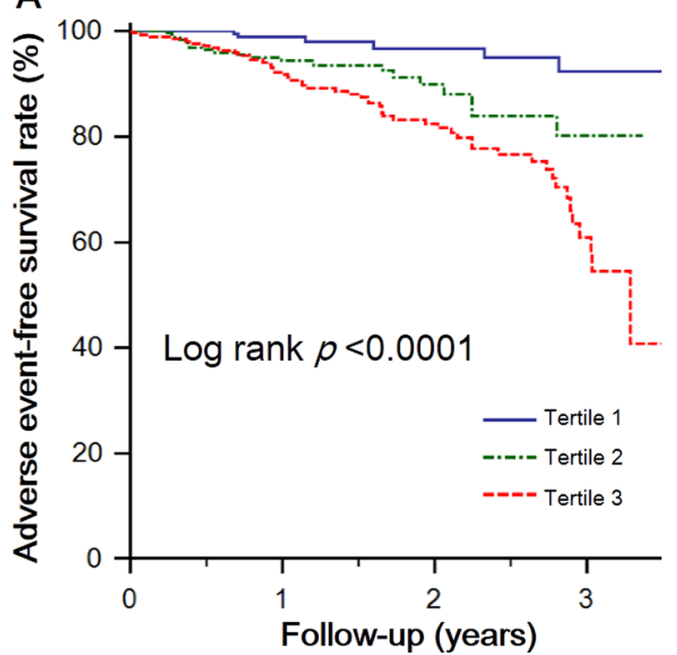

B

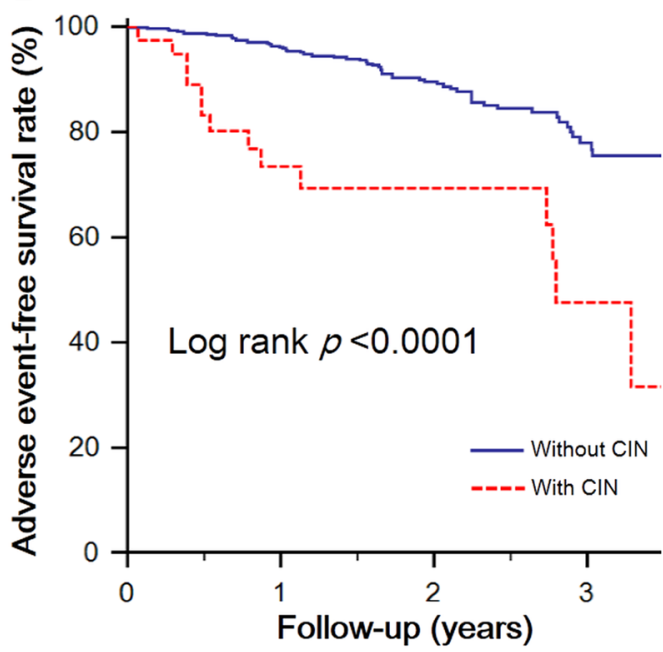

Figure 2. Kaplan-Meier curves of freedom from renal function decline events by (A) tertiles of the serum galectin-1 concentration and (B) the incidence of contrast-induced nephropathy.

$1.00-1.01 ; p=0.006)$ remained associated significantly with renal function decline (Table 4). Prior CIN was associated with renal function decline at a borderline significance level (HR, 1.98; 95\% CI, 0.96-4.09; $p=0.063$ ). The serum galectin-1 level was an independent predictor of renal function decline, rather than CIN, in patients undergoing CAG.

Subgroup analysis. The study cohort was stratified according to the presence of diabetes, presence of proteinuria, and PCI status. Increasing galectin- 1 concentrations were associated significantly with renal function decline, irrespective of underlying diseases, such as diabetes and proteinuria, or of PCI status ( $p_{\text {interaction }}>0.05$; Table 5).

\section{Discussion}

In this single-center observational study, the serum galectin-1 concentration was associated with eGFR decline during a mean follow-up period of $1.4 \pm 1.1$ years in 798 patients with stable angina undergoing elective CAG, irrespective of the presence of diabetes. To our knowledge, this longitudinal study is the first to explore the relationship between galectin-1 and subsequent renal function deterioration in patients undergoing CAG. These results demonstrate the involvement of galectin-1 in chronic renal function impairment, and suggest that the circulating galectin-1 level is an independent predictor of chronic renal function decline.

Much research attention has focused on galectin-1 and galectin-3, given their apparent major roles in cancer biology ${ }^{23}$. Recent reviews and meta-analyses have demonstrated that galectin-1 is associated with tumor formation, progression, metastasis, angiogenesis, and prognosis in several kinds of cancer ${ }^{23,24}$. Intracellularly, the galectin-1 protein provides a carbohydrate-independent scaffold for intracellular signaling pathways; extracellularly, it governs $\beta$-galactoside binding. Multivalent interactions between galectin- 1 and glycoproteins in the extracellular matrix contribute to cancer metastasis. A few in vitro and animal studies have mentioned the potential link between galectin-1 and kidney disease ${ }^{20-22,25-28}$, but no previous clinical report has described an association between the galectin-1 level and CKD.

The mechanisms linking galectin-1 elevation to CKD progression are not fully clear. Several groups have postulated the existence of mechanisms relating galectin-1 to $\mathrm{CKD}^{20}$, especially in the context of diabetes ${ }^{21,22}$. Increased galectin- 1 expression has been reported in diabetes ${ }^{19}$, diabetic retinopathy ${ }^{29}$ and diabetic nephropathy ${ }^{21,22}$. Galectin-1 has been shown to regulate podocin production and damage, and diabetic nephropathy progression, in podocytes ${ }^{22}$. In addition, recent evidence has revealed that galectin-1 is a fibrosis protein that is highly expressed in the kidneys of mice with types 1 and 2 diabetes, and activated in proximal tubular epithelial cells under high-glucose conditions ${ }^{22}$. The phosphorylation and activation of Akt also may play crucial roles in the modulation of activating enhancer binding protein 4 (AP4) to up-regulate the galectin-1 protein under hyperglycemic conditions in diabetic mice ${ }^{22}$. AP4 was identified as a protein binding to the galectin-1 promoter that regulates various functions under high glucose stimulation ${ }^{22}$.

Less is known about the association between galectin-1 and CKD in non-diabetic contexts. Emerging evidence suggests that the progression of cardiac and renal functions is often interconnected ${ }^{30,31}$. Cardiac and renal diseases have several common pathways, including those of enhanced systemic inflammation and stress-mediated neurohormonal responses, the development of anemia and bone and mineral disorders, and acid-base and fluid imbalances $^{31}$. Chronic myocardial dysfunction is also a well-known mediator of progressive renal function decline. The pathophysiology of this so-called type 2 cardiorenal syndrome remains under investigation ${ }^{30}$. Interestingly, a recent review suggested that galectin-1 is an emergent mediator of cardiovascular inflammation ${ }^{32}$. In this study, we also found that galectin-1 was associated with the LVEF and CAD in univariate analysis. We speculate that the parallel process affecting both heart and kidney functions also plays an essential role in the non-diabetic 


\begin{tabular}{|c|c|c|c|}
\hline Characteristic & Non-DM $(n=523)$ & $\mathrm{DM}(\mathrm{n}=275)$ & $P$ \\
\hline Age (years) & $65(58-76)$ & $70(60-78)$ & 0.003 \\
\hline Male, n (\%) & $344(65.8)$ & $195(70.9)$ & 0.152 \\
\hline Smoking, n (\%) & $168(32.1)$ & $97(35.3)$ & 0.385 \\
\hline $\mathrm{BMI}\left(\mathrm{kg} / \mathrm{m}^{2}\right)$ & $25.1(22.9-27.9)$ & $25.8(23.4-28.3)$ & 0.039 \\
\hline \multicolumn{4}{|l|}{ Medical history, $n(\%)$} \\
\hline Hypertension & $296(56.6)$ & $226(82.2)$ & $<0.001$ \\
\hline Chronic kidney disease & $14(2.7)$ & $35(12.7)$ & $<0.001$ \\
\hline Heart failure & $28(5.4)$ & $23(8.4)$ & 0.127 \\
\hline Peripheral arterial disease & $20(3.8)$ & $41(15.0)$ & $<0.001$ \\
\hline Previous stroke & $29(5.6)$ & $17(6.2)$ & 0.750 \\
\hline \multicolumn{4}{|l|}{ Medications, $n(\%)$} \\
\hline Antiplatelet agents & $254(48.6)$ & $170(61.8)$ & $<0.001$ \\
\hline ACEi/ARB & $128(24.5)$ & $105(38.2)$ & $<0.001$ \\
\hline Diuretics & $35(6.7)$ & $36(13.1)$ & 0.004 \\
\hline Oral antidiabetic agents & $0(0.0)$ & $148(53.8)$ & $<0.001$ \\
\hline Insulin & $0(0.0)$ & $45(16.4)$ & $<0.001$ \\
\hline Statins & $144(27.5)$ & $101(36.7)$ & 0.010 \\
\hline \multicolumn{4}{|l|}{ Laboratory data } \\
\hline White blood cells (K/cumm) & $6.6(5.6-7.6)$ & $7.0(5.7-8.2)$ & 0.007 \\
\hline Hemoglobin $(\mathrm{g} / \mathrm{dL})$ & $13.4(12.4-14.3)$ & $12.7(11.3-13.8)$ & $<0.001$ \\
\hline Fasting glucose $(\mathrm{mg} / \mathrm{dL})$ & $94(87-102)$ & $123(103-153)$ & $<0.001$ \\
\hline HbAlc (\%) & $5.9(5.6-6.1)$ & $7.1(6.5-8.0)$ & $<0.001$ \\
\hline Proteinuria (mg/dL) & $0.0(0.0-0.0)$ & $0.0(0.0-15.0)$ & $<0.001$ \\
\hline Proteinruia $\geq 30 \mathrm{mg} / \mathrm{dL}, \mathrm{n}(\%)$ & $24(2.7)$ & $54(19.9)$ & $<0.001$ \\
\hline eGFR $\left(\mathrm{ml} / \mathrm{min} / 1.73 \mathrm{~m}^{2}\right)$ & $72.7(59.8-85.7)$ & $64.5(44.8-80.8)$ & $<0.001$ \\
\hline Total cholesterol (mg/dL) & $165(143-186)$ & $152(132-175)$ & $<0.001$ \\
\hline Galectin 1 (ng/ml) & $17.0(14.2-23.0)$ & $20.5(15.9-28.5)$ & $<0.001$ \\
\hline \multicolumn{4}{|l|}{ Cardiac catheterization } \\
\hline Mean blood pressure $(\mathrm{mmHg})$ & $104(93-112)$ & $109.5(98-122)$ & 0.003 \\
\hline LV ejection fraction (\%) & $59.0(54.0-64.0)$ & $56.8(52.0-62.0)$ & 0.014 \\
\hline Significant CAD, n (\%) & $244(46.7)$ & $156(56.7)$ & 0.007 \\
\hline Underwent PCI, n (\%) & $210(40.2)$ & $145(52.7)$ & 0.001 \\
\hline Contrast volume $(\mathrm{ml})$ & $50.0(50.0-120.0)$ & $60.0(50.0-195.0)$ & $<0.001$ \\
\hline \multicolumn{4}{|l|}{ Outcomes } \\
\hline CIN, n (\%) & $18(3.4)$ & $23(8.4)$ & 0.004 \\
\hline eGFR decline $>30 \%, \mathrm{n}(\%)$ & $36(6.9)$ & $44(16.0)$ & $<0.001$ \\
\hline All-cause mortality, n (\%) & $7(1.3)$ & $6(2.2)$ & 0.388 \\
\hline
\end{tabular}

Table 2. Baseline characteristics of the study cohort according to the presence of diabetes mellitus. DM, diabetes mellitus; BMI, body mass index; $\mathrm{ACEi} / \mathrm{ARB}$, angiotensin-converting enzyme inhibitor/angiotensin receptor blocker; HbA1c, glycosylated hemoglobin A1c; eGFR, estimated glomerular filtration rate; LV, left ventricular; $\mathrm{CAD}$, coronary artery disease; $\mathrm{PCI}$, percutaneous coronary intervention; CIN, contrast-induced nephropathy.

association between galectin-1 and CKD. Our findings, in agreement with previous reports, illustrate the association of galectin-1 with renal function decline irrespective of diabetes. Galectin-1 thus may play a critical role in renal function decline in diabetic and non-diabetic patients.

Interestingly, we found no significant association between galectin-1 and CIN in patients undergoing CAG or PCI procedures. One possible explanation is that the mechanisms of CIN differ from those of chronic renal decline. Contrast media may alter nitric oxide, endothelin, and adenosine levels, inducing renal vasoconstriction and leading to renal medulla ischemia and acute tubular necrosis ${ }^{33,34}$. Contrast media may also have cytotoxic effects via the upregulation of reactive oxygen species ${ }^{33,35}$ or direct induction of osmotic tubular nephro$\operatorname{sis}^{36}$. Another possible reason is the lower incidence of CIN than renal function decline in our study sample. Furthermore, galectin- 1 and diabetes were correlated in this study and in previous reports ${ }^{17,18}$. Before adjustment for diabetes, tertile 3 of the galectin-1 level correlated with CIN. After adjustment for all related risk factors, including diabetes, this association was insignificant.

This study has some limitations that should be considered. First, the study population was relatively small, and consisted of Asian patients treated at a single hospital. Further studies larger numbers of different participants are required to confirm our findings. Second, patients enrolled in our study were elders (mean age, $67 \pm 12$ years). Caution should be taken when applying our findings to younger populations. Finally, the eGFR was used as the 


\begin{tabular}{|c|c|c|c|c|c|c|}
\hline \multirow[b]{2}{*}{ Outcome } & \multicolumn{2}{|l|}{ Univariable } & \multicolumn{2}{|l|}{ Model 1* } & \multicolumn{2}{|l|}{ Model $2^{\dagger}$} \\
\hline & Crude OR (95\% CI) & $P$ & Adjusted OR (95\% CI) & $P$ & Adjusted OR (95\% CI) & $P$ \\
\hline \multicolumn{7}{|l|}{ Galectin-1 } \\
\hline tertile 1 & Reference & & Reference & & Reference & \\
\hline tertile 2 & $1.75(0.68-4.51)$ & 0.248 & $1.54(0.59-4.02)$ & 0.381 & $1.80(0.67-4.81)$ & 0.244 \\
\hline tertile 3 & $3.34(1.40-7.95)$ & 0.007 & $2.64(1.03-6.76)$ & 0.044 & $2.04(0.77-5.43)$ & 0.152 \\
\hline Age & $1.04(1.01-1.07)$ & 0.003 & $1.04(1.00-1.07)$ & 0.034 & $1.04(1.01-1.08)$ & 0.026 \\
\hline Gender & $0.74(0.39-1.41)$ & 0.358 & $0.68(0.35-1.31)$ & 0.250 & $0.86(0.42-1.76)$ & 0.674 \\
\hline HTN & $1.68(0.81-3.48)$ & 0.163 & & & & \\
\hline Diabetes & $2.56(1.36-4.83)$ & 0.004 & & & $1.82(0.89-3.70)$ & 0.101 \\
\hline $\mathrm{Hb}$ & $0.63(0.53-0.75)$ & $<0.001$ & & & $0.67(0.54-0.83)$ & $<0.001$ \\
\hline eGFR & $0.98(0.97-1.00)$ & 0.033 & $1.00(0.98-1.02)$ & 0.853 & $1.02(1.00-1.04)$ & 0.062 \\
\hline Proteinuria & $1.01(1.00-1.01)$ & 0.004 & & & $1.00(1.00-1.01)$ & 0.057 \\
\hline LVEF & $0.99(0.96-1.02)$ & 0.457 & & & & \\
\hline Contrast & $1.00(1.00-1.01)$ & 0.150 & & & & \\
\hline
\end{tabular}

Table 3. Multivariable logistic regression analysis of the association between the serum galectin-1 concentration and the incidence of contrast-induced nephropathy. OR, odds ratio; CI, confidence interval; HTN, hypertension; Hb, hemoglobin; eGFR, estimated glomerular filtration rate; LVEF, left ventricular ejection fraction. *Adjusted for age, sex, and baseline eGFR; ${ }^{\dagger}$ adjusted for age, sex, and variables with $p$ values $<0.05$ in the univariable analysis.

\begin{tabular}{|c|c|c|c|c|c|c|}
\hline \multirow[b]{2}{*}{ Outcome } & \multicolumn{2}{|l|}{ Univariable } & \multicolumn{2}{|l|}{ Model 1* } & \multicolumn{2}{|l|}{ Model $2^{\dagger}$} \\
\hline & Crude HR (95\% CI) & $P$ & Adjusted HR (95\% CI) & $P$ & Adjusted HR (95\% CI) & $P$ \\
\hline \multicolumn{7}{|l|}{ Galectin-1 } \\
\hline tertile 1 & Reference & & Reference & & Reference & \\
\hline tertile 2 & $4.23(1.63-10.99)$ & 0.003 & $4.13(1.59-10.73)$ & 0.004 & $5.56(1.79-17.22)$ & 0.003 \\
\hline tertile 3 & $8.22(3.38-19.97)$ & $<0.001$ & $6.62(2.67-16.40)$ & $<0.001$ & $5.67(1.97-16.32)$ & 0.001 \\
\hline Age & $1.02(1.00-1.04)$ & 0.019 & $1.01(0.99-1.03)$ & 0.585 & $1.01(0.99-1.04)$ & 0.343 \\
\hline Gender & $1.00(0.63-1.60)$ & 0.997 & $0.98(0.61-1.57)$ & 0.926 & $0.91(0.53-1.58)$ & 0.746 \\
\hline HTN & $2.61(1.46-4.64)$ & 0.001 & & & $1.61(0.81-3.19)$ & 0.172 \\
\hline Diabetes & $2.43(1.56-3.78)$ & $<0.001$ & & & $1.31(0.75-2.31)$ & 0.343 \\
\hline $\mathrm{Hb}$ & $0.75(0.66-0.85)$ & $<0.001$ & & & $0.83(0.71-0.98)$ & 0.025 \\
\hline eGFR & $0.98(0.97-0.99)$ & $<0.001$ & $0.99(0.98-1.01)$ & 0.077 & $0.99(0.98-1.01)$ & 0.400 \\
\hline Proteinuria & $1.01(1.00-1.01)$ & $<0.001$ & & & $1.00(1.00-1.01)$ & 0.006 \\
\hline LVEF & $0.96(0.94-0.98)$ & $<0.001$ & & & $0.98(0.96-1.00)$ & 0.070 \\
\hline Prior CIN & $4.01(2.25-7.15)$ & $<0.001$ & & & $1.98(0.96-4.09)$ & 0.063 \\
\hline
\end{tabular}

Table 4. Multivariable Cox proportional-hazard analysis of the association of the serum galectin-1 concentration with the incidence of renal function decline. HR, hazard ratio; CI, confidence interval; HTN, hypertension; $\mathrm{Hb}$, hemoglobin; eGFR, estimated glomerular filtration rate; LVEF, left ventricular ejection fraction; CIN, contrast-induced nephropathy. *Adjusted for age, sex, and baseline eGFR; ${ }^{\dagger}$ adjusted for age, sex, and variables with $p$ values $<0.05$ in the univariable analysis.

sole renal function endpoint, without consideration of other clinical endpoints affecting renal outcomes, including newly diagnosed diabetes mellitus and the progression of proteinuria. Nevertheless, our study demonstrated that the serum galectin-1 level is a novel risk marker for renal outcomes in patients with suspected CAD undergoing CAG or PCI procedures.

\section{Conclusions}

Although not a predictor of CIN, the circulating serum galectin-1 level is an independent prognostic marker for subsequent renal function decline in patients undergoing CAG, irrespective of diabetes. These findings provide novel evidence of galectin-1's involvement in the pathogenesis of renal dysfunction in patients with suspected CAD. Further research exploring the underlying mechanism is needed.

\section{Methods}

Study design and patient population. A team of cardiologists (under the direction of Prof. Po-Hsun Huang) collected data for this study consecutively from June 2011 to August 2018. The sample size was not pre-calculated. In total, 855 subjects with stable coronary CAD admitted to Taipei Veterans General Hospital for elective CAG and/or PCI were screened. Each patient's serum creatinine concentration was measured before CAG, and the eGFR was calculated using the CKD Epidemiology Collaboration equation ${ }^{37}$. Patients with stage 5 CKD, defined 


\begin{tabular}{|c|c|c|c|}
\hline \multirow{2}{*}{$\begin{array}{l}\text { Subgroup } \\
\text { (events/subjects) }\end{array}$} & \multicolumn{2}{|l|}{ Galectin-1 } & \multirow[b]{2}{*}{$P$ for interaction } \\
\hline & Adjusted HR (95\% CI) & $P$ & \\
\hline \multicolumn{4}{|l|}{ Overall (80/798) } \\
\hline tertile 2 & $5.56(1.79-17.22)$ & 0.003 & \\
\hline tertile 3 & $5.67(1.97-16.32)$ & 0.001 & \\
\hline \multicolumn{4}{|c|}{ With diabetes $(44 / 275)$} \\
\hline tertile 2 & $3.88(0.92-16.41)$ & 0.065 & 0.236 \\
\hline tertile 3 & $3.55(0.99-12.81)$ & 0.053 & \\
\hline \multicolumn{4}{|c|}{ Without diabetes $(36 / 523)$} \\
\hline tertile 2 & $10.45(1.27-85.77)$ & 0.029 & \\
\hline tertile 3 & $12.11(1.57-93.40)$ & 0.017 & \\
\hline \multicolumn{4}{|c|}{ With proteinuria $(42 / 78)$} \\
\hline tertile 2 & $7.57(0.76-75.56)$ & 0.085 & 0.560 \\
\hline tertile 3 & $9.61(1.17-79.11)$ & 0.035 & \\
\hline \multicolumn{4}{|c|}{ Without proteinuria $(38 / 720)$} \\
\hline tertile 2 & $3.90(1.04-14.62)$ & 0.043 & \\
\hline tertile 3 & $3.72(1.07-13.02)$ & 0.039 & \\
\hline \multicolumn{4}{|c|}{ Underwent PCI (39/355) } \\
\hline tertile 2 & $9.32(1.12-77.44)$ & 0.039 & 0.115 \\
\hline tertile 3 & $8.51(1.06-68.23)$ & 0.044 & \\
\hline \multicolumn{4}{|c|}{ Not underwent PCI (41/443) } \\
\hline tertile 2 & $2.08(0.38-11.52)$ & 0.400 & \\
\hline tertile 3 & $3.13(0.84-11.67)$ & 0.089 & \\
\hline
\end{tabular}

Table 5. Stratified analysis of the effect of the serum galectin-1 concentration on renal function decline in patients grouped by diabetes, proteinuria, and percutaneous coronary intervention status. HR, hazard ratio; CI, confidence interval; PCI: percutaneous coronary intervention. *Adjusted for age, sex, hypertension, diabetes, hemoglobin, baseline estimated glomerular filtration rate, proteinuria, left ventricular ejection fraction, and prior contrast-induced nephropathy.

as creatinine clearance $<15 \mathrm{ml} / \mathrm{min} / 1.73 \mathrm{~m}^{2}(n=15)$, and those undergoing hemodialysis or peritoneal dialysis $(n=42)$ were excluded from the analysis. Thus, a total of 798 patients was enrolled in the study and included in the final analysis (Fig. 1). The patients were grouped into tertiles according to the serum galectin-1 concentration.

Each patient's chart was reviewed in detail to collect data on medications, smoking status, and risk factors for CIN, such as age, existing renal dysfunction, type 2 diabetes mellitus, and volume depletion. Patients' blood pressure was measured using electronic sphygmomanometers at least four times a day during hospitalization. Hypertension was defined as systolic blood pressure $\geq 140 \mathrm{mmHg}$, diastolic blood pressure $\geq 90 \mathrm{mmHg}$, or the use of antihypertensive medications. Type 2 diabetes mellitus was defined as fasting plasma glucose level $\geq 126 \mathrm{mg} / \mathrm{dl}$ or the use of hypoglycemic agents. The body mass index was calculated by dividing the weight of the patient (in kilograms) by the square of the height (in meters). A nonionic low-osmolality contrast medium (iopromide) was used for all patients' examinations. It was administered intra-arterially, mainly through transradial catheters. Metformin and nephrotoxic medications, such as non-steroidal anti-inflammatory drugs, were discontinued $48 \mathrm{~h}$ before contrast medium administration. Before and after contrast medium exposure, physiological (0.9\%) saline was given intravenously at a rate of $1 \mathrm{ml} / \mathrm{kg} / \mathrm{h}$ for $12 \mathrm{~h}$. In patients with left ventricular dysfunction (ejection fraction $<40 \%$ ) or apparent heart failure, the hydration rate was reduced to $0.5 \mathrm{ml} / \mathrm{kg} / \mathrm{h}$. This research was conducted according to the principles expressed in the Declaration of Helsinki. This study was approved by the research ethics committee of Taipei Veterans General Hospital, and all participants provided written informed consent.

Laboratory investigations and cardiac catheterization. Blood samples were collected after an $\geq 8$-h fast. The blood cell count; serum glucose, creatinine, and uric acid levels; and lipid profiles were determined using a Hitachi 7600 autoanalyzer (Hitachi Ltd., Tokyo, Japan). The serum creatinine concentration was assessed at the time of admission and daily for the following 3 days after contrast medium exposure. Urine dipstick analysis was performed using commercial test strips, and proteinuria was defined as a urine protein concentration $\geq 30 \mathrm{mg} / 100 \mathrm{ml}$. Plasma concentrations of galectin-1 were determined using the commercially available Human Galectin-1 Quantikine ELISA Kit DGAL10 (R\&D Systems, Inc., Minneapolis, MN, USA); the sensitivity was $0.129 \mathrm{ng} / \mathrm{ml}$ and the assay range was $0.3-20 \mathrm{ng} / \mathrm{ml}$. The intra- and interassay coefficients were $5.7-8.8 \%$ and $7.5-9.5 \%$, respectively. Two experienced interventional cardiologists interpreted the coronary angiograms. Coronary lesions causing $>50 \%$ diameter narrowing were considered to represent significant stenosis. The LVEF was estimated by left ventriculography. The contrast consumption of each patient was also recorded.

Definition of study endpoints and renal function decline. All patients were evaluated for the occurrence of CIN, which was defined as an increase in the serum creatinine concentration of $\geq 0.5 \mathrm{mg} / \mathrm{dl}$ or a $25 \%$ increase from baseline within $48 \mathrm{~h}$ after $\mathrm{CAG}^{38}$. Patients were advised to visit outpatient clinics regularly after 
discharge from the hospital. The cohort was followed until January 2019 or the occurrence of death. Patients' clinical data, including serum creatinine levels, were obtained every 3-6 months during the follow-up period. Renal function decline was defined as a $>30 \%$ decrease in the eGFR after discharge ${ }^{39,40}$. When it was identified, the serum creatinine level was re-measured 1 month later to ensure the accuracy of the diagnosis.

Statistical analysis. Data were expressed as medians (interquartile ranges) for numeric variables and as numbers (percentages) for categorical variables. Clinical and laboratory data were compared using the MannWhitney U test for continuous variables and Fisher's exact test for categorical variables. The incidences of CIN and renal function decline were calculated. Survival curves were generated using the Kaplan-Meier method, and survival was compared among groups using the log-rank test. Logistic regression analysis was performed to examine the relationships of various risk factors to CIN, and Cox proportional-hazard regression analysis was performed to identify risk factors for progressive renal function decline. Factors that were significant in univariate regression analysis were entered into the multivariable regression analysis. To investigate modification of the effect of galectin-1 on renal function decline by different comorbidities, we performed subgroup analyses with the study cohort stratified according to the presence of diabetes, presence of proteinuria, and PCI status. Interactions between the galectin-1 concentration and comorbid conditions were examined by adding a product term of galectin-1 and comorbidities to the Cox regression analysis. Data were analyzed using SPSS version 18.0 (SPSS Inc., Chicago, IL). P values $<0.05$ were regarded as significant.

Data availability. The datasets generated and/or analyzed during the current study are available from the corresponding author on reasonable request.

Received: 12 August 2019; Accepted: 5 January 2020;

Published online: 29 January 2020

\section{References}

1. Levey, A. S. et al. National Kidney Foundation practice guidelines for chronic kidney disease: evaluation, classification, and stratification. Ann. Intern. Med. 139, 137-147, https://doi.org/10.7326/0003-4819-139-2-200307150-00013 (2003).

2. Sachdev, M. et al. The prognostic importance of comorbidity for mortality in patients with stable coronary artery disease. J. Am. Coll. Cardiol. 43, 576-582, https://doi.org/10.1016/j.jacc.2003.10.031 (2004).

3. Eng, J. et al. Comparative effect of contrast media type on the incidence of contrast-induced nephropathy: a systematic review and meta-analysis. Ann. Intern. Med. 164, 417-424, https://doi.org/10.7326/M15-1402 (2016).

4. McCullough, P. A. M. D. et al. Acute renal failure after coronary intervention: incidence, risk factors, and relationship to mortality. Am. J. Med. 103, 368-375, https://doi.org/10.1016/S0002-9343(97)00150-2 (1997).

5. Maioli, M. et al. Persistent renal damage after contrast-induced acute kidney injury: incidence, evolution, risk factors, and prognosis. Circulation 125, 3099-3107, https://doi.org/10.1161/CIRCULATIONAHA.111.085290 (2012).

6. James, M. T. et al. Acute kidney injury following coronary angiography is associated with a long-term decline in kidney function. Kidney Int. 78, 803-809, https://doi.org/10.1038/ki.2010.258 (2010).

7. Mehran, R. et al. A simple risk score for prediction of contrast-induced nephropathy after percutaneous coronary intervention. $J$. Am. Coll. Cardiol. 44, 1393-1399, https://doi.org/10.1016/j.jacc.2004.06.068 (2004).

8. Wybraniec, M. T., Bożentowicz-Wikarek, M., Chudek, J. \& Mizia-Stec, K. Pre-procedural renal resistive index accurately predicts contrast-induced acute kidney injury in patients with preserved renal function submitted to coronary angiography. Int. J. Cardiovasc. Imaging 33, 595-604, https://doi.org/10.1007/s10554-016-1039-1 (2016).

9. Watanabe, M. et al. Prediction of contrast-induced nephropathy by the serum creatinine level on the day following cardiac catheterization. J. Cardiol. 68, 412-418, https://doi.org/10.1016/j.jjcc.2015.10.016 (2016).

10. Chaykovska, L. et al. Urinary vitamin D binding protein and KIM-1 are potent new biomarkers of major adverse renal events in patients undergoing coronary angiography. PLoS One 11, e0145723, https://doi.org/10.1371/journal.pone.0145723 (2016).

11. Torregrosa, I. et al. Urinary KIM-1, NGAL and L-FABP for the diagnosis of AKI in patients with acute coronary syndrome or heart failure undergoing coronary angiography. Heart Vessel. 30, 703-711, https://doi.org/10.1007/s00380-014-0538-z (2015).

12. Akdeniz, D. et al. Is kidney injury molecule 1 a valuable tool for the early diagnosis of contrast-induced nephropathy? J. Investig. Med. 63, 930-934, https://doi.org/10.1097/JIM.0000000000000243 (2015).

13. Junrong Tong, H. L. et al. Neutrophil gelatinase-associated lipocalin in the prediction of contrast-induced nephropathy: a systemic review and meta-analysis. J. Cardiovasc. Pharmacol. 66, 239-245 (2015).

14. Briguori, C. et al. Cystatin C and contrast-induced acute kidney injury. Circulation 121, 2117-2122, https://doi.org/10.1161/ CIRCULATIONAHA.109.919639 (2010).

15. Barondes, S. H., Cooper, D. N., Gitt, M. A. \& Leffler, H. Galectins: structure and function of a large family of animal lectins. J. Biol. Chem. 269, 20807-20810 (1994).

16. Camby, I., Le Mercier, M., Lefranc, F. \& Kiss, R. Galectin-1: a small protein with major functions. Glycobiol. 16, 137R-157R, https:// doi.org/10.1093/glycob/cwl025 (2006).

17. Tsai, W.-C. et al. Risk factors for development and progression of chronic kidney disease. Med. 95, e3013, https://doi.org/10.1097/ md.0000000000003013 (2016).

18. Fryk, E. et al. Microdialysis and proteomics of subcutaneous interstitial fluid reveals increased galectin-1 in type 2 diabetes patients. Metab. 65, 998-1006, https://doi.org/10.1016/j.metabol.2016.04.003 (2016).

19. Liu, X. et al. Proteomics-based identification of differentially-expressed proteins including galectin-1 in the blood plasma of type 2 diabetic patients. J. Proteome Res. 8, 1255-1262, https://doi.org/10.1021/pr800850a (2009).

20. Potthoff, S. A. et al. The glomerular proteome in a model of chronic kidney disease. Proteom. Clin. Appl. 2, 1127-1139, https://doi. org/10.1002/prca.200800010 (2008).

21. Liu, Y. et al. High glucose-induced Galectin-1 in human podocytes implicates the involvement of Galectin-1 in diabetic nephropathy. Cell Biol. Int. 39, 217-223, https://doi.org/10.1002/cbin.10363 (2015).

22. Al-Obaidi, N. et al. Galectin-1 is a new fibrosis protein in type 1 and type 2 diabetes. FASEB J. 33, 373-387, https://doi.org/10.1096/ fj.201800555RR (2019).

23. Cousin, J. M. \& Cloninger, M. J. The role of galectin-1 in cancer progression, and synthetic multivalent systems for the study of galectin-1. Int. J. Mol. Sci. 17, E1566, https://doi.org/10.3390/ijms17091566 (2016).

24. Huang, M. Y., He, J. P., Zhang, W. Q. \& Liu, J. L. Pooling analysis reveals that galectin-1 is a reliable prognostic biomarker in various cancers. J. Cell Physiol. 234, 13788-13798, https://doi.org/10.1002/jcp.28059 (2019). 
25. Valkova, N., Yunis, R., Mak, S. K., Kang, K. \& Kültz, D. Nek8 mutation causes overexpression of galectin-1, sorcin, and vimentin and accumulation of the major urinary protein in renal cysts of jck mice. Mol. Cell. Proteom. 4, 1009-1018 (2005).

26. Ostalska-Nowicka, D. et al. Immunohistochemical detection of galectin-1 in renal biopsy specimens of children and its possible role in proteinuric glomerulopathies. Histopathology 51, 468-476, https://doi.org/10.1111/j.1365-2559.2007.02818.x (2007).

27. Shimizu, M. et al. Expression of galectin-1, a new component of slit diaphragm, is altered in minimal change nephrotic syndrome. Lab. Invest. 89, 178-195, https://doi.org/10.1038/labinvest.2008.125 (2009).

28. Okano, K. et al. Suppression of renal fibrosis by galectin-1 in high glucose-treated renal epithelial cells. Exp. Cell Res. 316, 3282-3291, https://doi.org/10.1016/j.yexcr.2010.08.015 (2010).

29. Kanda, A., Dong, Y., Noda, K., Saito, W. \& Ishida, S. Advanced glycation endproducts link inflammatory cues to upregulation of galectin-1 in diabetic retinopathy. Sci Rep 7, https://doi.org/10.1038/s41598-017-16499-8 (2017).

30. Ronco, C., Haapio, M., House, A. A., Anavekar, N. \& Bellomo, R. Cardiorenal syndrome. J. Am. Coll. Cardiol. 52, 1527-1539, https:// doi.org/10.1016/j.jacc.2008.07.051 (2008).

31. Schefold, J. C., Filippatos, G., Hasenfuss, G., Anker, S. D. \& von Haehling, S. Heart failure and kidney dysfunction: epidemiology, mechanisms and management. Nat. Rev. Nephrol. 12, 610, https://doi.org/10.1038/nrneph.2016.113 (2016).

32. Seropian, I. M. et al. Galectin-1 as an emerging mediator of cardiovascular inflammation: mechanisms and therapeutic opportunities. Mediators Inflamm 2018, 1-11, https://doi.org/10.1155/2018/8696543 (2018)

33. Persson, P. B., Hansell, P. \& Liss, P. Pathophysiology of contrast medium-induced nephropathy. Kidney Int. 68, 14-22, https://doi. org/10.1111/j.1523-1755.2005.00377.x (2005).

34. Weisberg, L. S., Kurnik, P. B. \& Kurnik, B. R. Radiocontrast-induced nephropathy in humans: role of renal vasoconstriction. Kidney Int. 41, 1408-1415 (1992).

35. Heinrich, M. C. et al. Cytotoxic effects of ionic high-osmolar, nonionic monomeric, and nonionic iso-osmolar dimeric iodinated contrast media on renal tubular cells in vitro. Radiology 235, 843-849, https://doi.org/10.1148/radiol.2353040726 (2005).

36. Azzalini, L., Spagnoli, V. \& Ly, H. Q. Contrast-induced nephropathy: from pathophysiology to preventive strategies. Can. J. Cardiol. 32, 247-255, https://doi.org/10.1016/j.cjca.2015.05.013 (2016).

37. Levey, A. S. et al. A new equation to estimate glomerular filtration rate development and comparison of a new equation to estimate GFR. Ann. Intern. Med. 150, 604-612, https://doi.org/10.7326/0003-4819-150-9-200905050-00006 (2009).

38. McCullough, P. A. Contrast-induced acute kidney injury. J. Am. Coll. Cardiol. 51, 1419-1428, https://doi.org/10.1016/j. jacc.2007.12.035 (2008).

39. Levey, A. S. et al. GFR decline as an end point for clinical trials in CKD: a scientific workshop sponsored by the National Kidney Foundation and the US Food and Drug Administration. Am. J. Kidney Dis. 64, 821-835, https://doi.org/10.1053/j.ajkd.2014.07.030 (2014).

40. Wu, C. H. et al. Circulating fibroblast growth factor 21 is associated with subsequent renal injury events in patients undergoing coronary angiography. Sci. Rep. 8, 12425, https://doi.org/10.1038/s41598-018-30744-8 (2018).

\section{Acknowledgements}

This study was supported, in part, by research grants from the Ministry of Science and Technology of Taiwan (MOST 106-2314-B-350-001-MY3); the Novel Bioengineering and Technological Approaches to Solve Two Major Health Problems in Taiwan program, sponsored by the Taiwan Ministry of Science and Technology Academic Excellence Program (MOST 108-2633-B-009-001); the Ministry of Health and Welfare (MOHW106TDU-B-211-113001); and Taipei Veterans General Hospital (V105C-207, V106C-045, V108C-195). These funding agencies had no influence on the study design, data collection or analysis, the decision to publish, or the preparation of the manuscript.

\section{Author contributions}

Study concept and design: C.-S.K., P.-H.H., and S.-J.L.; acquisition of data: P.-H.H., and S.-J.L.; analysis and interpretation of data: R.-H.C., C.-S.K., Y.-W.L., and Y.-L.T.; drafting of the manuscript: C.-S.K., R.-H.C., and P.-H.H.; statistical analysis: R.-H.C., Y.-W.L., and Y.-L.T.; administrative, technical, or material support: C.-S.K., P.-H.H., and S.-J.L.; critical revision: C.-S.K., P.-H.H., and S.-J.L.; study supervision: P.-H.H., and S.-J.L. The corresponding authors have the right to grant on behalf of all authors and do grant on behalf of all authors a worldwide license to the publisher and its licensees in perpetuity.

\section{Competing interests}

The authors declare no competing interests.

\section{Additional information}

Correspondence and requests for materials should be addressed to P.-H.H. or S.-J.L.

Reprints and permissions information is available at www.nature.com/reprints.

Publisher's note Springer Nature remains neutral with regard to jurisdictional claims in published maps and institutional affiliations.

Open Access This article is licensed under a Creative Commons Attribution 4.0 International License, which permits use, sharing, adaptation, distribution and reproduction in any medium or format, as long as you give appropriate credit to the original author(s) and the source, provide a link to the Creative Commons license, and indicate if changes were made. The images or other third party material in this article are included in the article's Creative Commons license, unless indicated otherwise in a credit line to the material. If material is not included in the article's Creative Commons license and your intended use is not permitted by statutory regulation or exceeds the permitted use, you will need to obtain permission directly from the copyright holder. To view a copy of this license, visit http://creativecommons.org/licenses/by/4.0/.

(c) The Author(s) 2020 